\title{
The future of emergency medicine in Canada: Reflections one year after the release of the Collaborative Working Group final report
}

\author{
Paul Pageau, MD*; Douglas Sinclair, $\mathrm{MD}^{\dagger}$; Riyad B. Abu-Laban, MD, MHSc ${ }^{\ddagger}$; Pamela Eisener- \\ Parsche, $\mathrm{MD}^{\S}$; Jason Frank, MD, MA(Ed)*; Brian R. Holroyd, MD, MBA ${ }^{\text {Il; }}$ Constance LeBlanc, MD, \\ $\mathrm{MA}(\mathrm{Ed})^{\|} ;$Peter Toth, MD, MSc ${ }^{* *}$
}

On June 4, 2016, at the Canadian Association of Emergency Physicians (CAEP) annual meeting in Quebec City, the final report of the Collaborative Working Group (CWG) on the Future of Emergency Medicine in Canada was presented. This work represented the culmination of an unprecedented trilateral initiative that commenced in 2013 and involved balanced representation from the CAEP, the College of Family Physicians of Canada (CFPC), and the Royal College of Physician and Surgeons of Canada (RCPSC).

The CWG used a number of methods to capture data including a review of all literature and prior initiatives, an environmental scan, and a detailed comparative analysis of the two Canadian Emergency Medicine (EM) training programs. These data were combined with information obtained from communication with all university postgraduate deans of medical education and key stakeholders. An extensive mixed methods national survey was also conducted of emergency department (ED) chiefs, emergency physicians with EM certification from either College, residents from both EM training programs, and CFPC-certified physicians with no EM certification who indicated that they had an interest or activity in EM practice. The resulting report was significant in both its breadth and depth and is available on the CAEP website (http://caep.ca/about/about/collaborative-working-groupfinal-report). We feel it is required reading for all physicians involved in EM in Canada.
One of the most remarkable findings of the CWG was the identification of a significant human health resource (HHR) shortfall of emergency physicians in Canada that is currently estimated at 478 physicians and predicted to rise to 1,071 physicians by 2020 and 1,518 physicians by 2025 , in the absence of any expansion of EM training programs.

The CWG did not recommend a single certification stream for EM practice but noted in the report that both Colleges should remain aware that substantial support exists in the EM community for this, as does a level of dissatisfaction among the residents in both EM programs that is higher than documented in other disciplines. Meaningful improvements to the status quo are required; in that context, the CWG made a number of specific recommendations to both Colleges regarding both the makeup of the Certificate of the College Family Physicians - EM (CCFP-EM) and RCPSC programs and their future coordination and collaboration.

The provision of EM care in rural Canada, where the majority of EM care is delivered by physicians without EM certification, emerged as a key consideration. While a fulsome analysis of this aspect was outside qthe terms of reference of the CWG, the final report did recommend that the CFPC, the Society of Rural Physicians of Canada, and other key stakeholders work collaboratively toward solutions in this important area.

\footnotetext{
From the *Department of Emergency Medicine, University of Ottawa, Ottawa, ON; tDepartment of Emergency Medicine, St. Michael's Hospital, Toronto, ON; ¥Department of Emergency Medicine, Vancouver General Hospital, Vancouver, BC; §Department of Family Medicine, University of Ottawa and Bruyere Continuing Care, Ottawa, ON; ףDepartment of Emergency Medicine, University of Alberta, Edmonton, AB; IDepartment of Emergency Medicine, Dalhousie University, Halifax, NS; and the ${ }^{* *}$ Department of Family and Community Medicine, University of Toronto, Toronto, ON.
}

Correspondence to: Dr. Paul Pageau, The Ottawa Hospital, 1053 Carling Ave, Ottawa ON K1Y 4E9; Email: ppageau@toh.ca 
One year later, the CWG report continues to be discussed; some actions have been taken, but there is much more to do.

On the HHR issue, there has been an interest in looking at specific regional HHR needs in Ontario, BC, and Atlantic Canada, and analyses are ongoing. Decisions regarding postgraduate training positions in each province are made by the Ministries of Health and postgraduate deans of the medical schools. Neither the Royal College nor the CFPC advocate for a specific number of training positions, only that the standards for each training program be met. In Ontario, Dr. Howard Ovens, Ontario expert lead for EM, is leading a provincial analysis of the HHR data, to develop the case for expanding EM training programs. Each province/ region will require similar local leadership to move this agenda forward.

The Royal College is currently developing its Competence by Design project, which the CWG believes should address some of the recommendations and concerns raised in its report. Dr. Jonathan Sherbino, the chair of the Royal College EM Specialty Committee, is the lead on this revision of the Royal College EM training program. Recently, the CFPC was formally approached to provide a representative to collaborate on this work.

The CFPC-EM Practice Committee, chaired by Dr. John Foote, has endorsed all the recommendations in the CWG report. An action plan by the CFPC to review both the content and structure of the CCFP-EM program and CCFP program as it relates to Emergency Medicine is now under development. The CFPC is also in active discussions with the Society of Rural Physicians of Canada to delineate issues regarding EM care in rural Canada.

The survey results of the CWG remain rich data sets, available to policy makers and researchers to utilize for the purposes of improving EM care in Canada. The CWG developed a list of potential research questions that could be addressed with the survey data set, and the report encouraged researchers interested in this topic to consider pursuing these or other topics.

CAEP remains essential to advancing the CWG recommendations and the future of EM in Canada. Although the CAEP board of directors strongly endorsed the CWG recommendations, CAEP does not have a mandate or role in the development or certification of EM training programs. However, CAEP is the key advocate for EM at multiple levels across Canada. To this end, CAEP recently established a new "Future of Emergency Medicine" committee, which will report to the CAEP board. This new committee will work in a leadership and advocacy capacity to enact the CWG recommendations. It will develop a strategy to help CAEP maintain a leadership role in facilitating communication and collaboration among the Colleges, postgraduate deans, and provincial government decision makers. Addressing the HHR shortfall and finding solutions to this problem is particularly important to CAEP members and will continue to be a strategic priority for CAEP. The first meeting of the "Future of Emergency Medicine Committee" was held at the CAEP conference in June 2017.

It is important to note that the recommendations made by the CWG were not a judgment on the current variation in practice experience and certification across the nation but rather represent a collective vision for the future based on a rigorous process that included the first comprehensive survey of the entire Canadian EM community. The wheels of change turn slowly on issues of this magnitude, but they do appear to be slowly turning. One year later, there remains much to implement and improve. As a reminder from the late Nelson Mandela, "It always seems impossible . . . until it is done." 